\title{
Influencia de la Fertilización en la Calidad del Suelo de Cultivo de Maiz - Caso Loreto
}

\author{
Marcela Yolanda Brito Mancero, \\ Facultad de Ciencias de la Escuela Superior Politécnica de Chimborazo, \\ Magister en Agricultura Sostenible \\ Lourdes Cumanda Carrera Beltran,
}

Facultad de Ciencias de la Escuela Superior Politécnica de Chimborazo,

Master Universitario en Ingeniería para el Ambiente y el Territorio

Luis Miguel Santillán Quiroga,

Facultad de Ciencias de la Escuela Superior Politécnica de Chimborazo,

Magister en Protección Ambiental

\section{Resumen}

El suelo es uno de los recursos indispensables para el desarrollo de la vida, ya que este se convierte en una pieza clave en el ciclo natural de materia y energía. Cuando se habla del suelo en la agricultura debe cumplir condiciones específicas para la producción vegetal, conocido como "suelo fértil". Esta definición se centra tanto en las condiciones físicas, químicas, biológicas ideales como en la disponibilidad de nutrientes que el suelo presenta para un adecuado desarrollo de las especies que se desea obtener. Sin embargo, este criterio es muy limitado, es necesario reconocer todas las funciones de este recurso y la importancia que tienen para la sostenibilidad del ambiente y la creciente necesidad de producción de alimentos más sanos. El evaluar los cambios del suelo mediante diferentes indicadores edáficos y de manejo se convierte en un instrumento que permite valorar la calidad ambiental del recurso y proporciona información para tomar las medidas necesarias que permitan su conservación y el mejoramiento de la producción agrícola. El presente articulo pretende contribuir en la toma de decisiones para el manejo del cultivo de maíz en el cantón Loreto, utilizando como instrumento el estudio del suelo actual mediante el análisis de la repercusión de diferentes tipos de fertilización del cultivo de maíz en nueve indicadores edáficos que son: temperatura, $\mathrm{pH}$, infiltración, materia orgánica, nitrógeno amoniacal, fósforo, potasio, calcio y magnesio, así como las repercusiones que tiene el uso de diferentes fertilizantes en los índices de calidad. 
Palabras Clave: Indicadores de calidad, influencia de fertilización, calidad de suelo, cultivo de maíz, suelo fértil, macronutrientes

\title{
Influence of Fertilization on the Quality of Corn Cultivation Soil: A Case Study of Loreto
}

\author{
Marcela Yolanda Brito Mancero, \\ Facultad de Ciencias de la Escuela Superior Politécnica de Chimborazo, \\ Magister en Agricultura Sostenible \\ Lourdes Cumanda Carrera Beltran,
}

Facultad de Ciencias de la Escuela Superior Politécnica de Chimborazo, Master Universitario en Ingeniería para el Ambiente y el Territorio

Luis Miguel Santillán Quiroga,

Facultad de Ciencias de la Escuela Superior Politécnica de Chimborazo, Magister en Protección Ambiental

\begin{abstract}
One of the indispensable natural resources for the development of life is the soil. This is because it is a key piece in the natural cycle of matter and energy. When we talk about soil in agriculture, we are making reference to "fertile soil" which must meet specific conditions for plant production. This definition focuses on the physical and chemical conditions and the availability of nutrients that the soil presents for an adequate development of the species which an individual desires to get. However, this criterion is very limited. It is also necessary to recognize all the functions of this resource and the significant role they play in the sustainability of the environment as well as the growing need for the production of healthier foods. The evaluation of soil changes, by means of different edaphic indicators and management, has become a necessary tool to assess the quality of the resource. It also provides information to be able to take the necessary measures to allow soil conservation and improve agricultural production at the same time. This paper focuses on contributing to decision making in the management of maize cultivation in the Loreto canton. The instrument used is the knowledge of the current state of the soil, and this is done through the analysis of nine indicators which include: temperature, $\mathrm{pH}$, infiltration, organic matter, ammonia nitrogen, phosphorus, potassium, calcium, and magnesium. The repercussions that the use of different fertilizers has on the quality indexes were also discussed.
\end{abstract}


Keywords: Indicators of quality, influence of fertilization, soil quality, corn cultivation fertile soil, macronutrients

\section{Introduction}

El suelo es considerado como un eslabón en el ciclo natural de materia y energía por lo que se le reconoce como un sistema vivo y dinámico que funciona a través del equilibrio e interacción de los componentes biológicos, químicos y físicos que lo constituyen.

García (2012) menciona que la "calidad del suelo" se comenzó a utilizar cuando se reconocieron las diferentes funciones en el desarrollo de la productividad del sistema sin perturbar sus propiedades físicas, químicas y biológicas; aminorar los contaminantes; y, beneficiar la salud de los seres vivos. Por lo que (Cantú, 2007) propone que los suelos que se encuentren en escala de 0,80 a 1,00 se les calificará como suelos de muy alta calidad; de 0,60 a 0,7 son de alta calidad, de 0,40 a 0,59 moderada calidad, y 0,00 a 0,19 se le califica como muy baja calidad.

El Comité para la Salud del suelo de la Soil Science Society of America sintetizó esta definición como, la capacidad del suelo para funcionar dentro de los límites de un ecosistema natural o manejado (Astier-Calderón, 2002). Para que este concepto sea práctico es preciso contar con datos que permitan evaluar la condición del suelo. Por lo que surgen los indicadores, que son variables que representan una condición y muestran información sobre los cambios o tendencias (Dumanski, 1998). Los indicadores de calidad del suelo, se crean como una herramienta de medición que ofrece información sobre las propiedades, procesos y características; los mismos que se miden para dar seguimiento a los efectos del manejo y sobre el funcionamiento del suelo en un determinado período (Astier-Calderón, 2002). La calidad del suelo es considerada como una medida de su capacidad para funcionar adecuadamente en relación con un uso específico (Gregorich, 1994). Conocer la calidad de los suelos es de suma importancia ya que contribuyen a establecer la sostenibilidad de las diferentes actividades que se llevan a cabo en él (Moreno, 2015). Los suelos con máxima calidad son capaces de mantener alta productividad y causar el mínimo deterioro ambiental (Ferreras, et al., 2009).

Para definir la fertilidad del suelo se han propuesto variados conceptos; la definición más común de suelo fértil plantea que es aquel que tiene la capacidad de aprovisionar la cantidad suficiente de nutrientes al cultivo para asegurar un crecimiento y desarrollo deseado (Havlin, 1999). Dicha definición se replantea una vez que la agricultura industrial empieza a mostrar un impacto negativo en el suelo (Soto, 2006). En el Ecuador según el lineamiento que maneja Agrocalidad, (Moreno, 2015), menciona al índice de fertilidad como un factor para la evaluación de la calidad de suelo. 
El cultivo de maíz es una las principales actividades agrícolas que realizan las Comunidades Kichwas de Orellana como fuentes generadoras de ingresos, actividades que realizan de manera tradicional en todo el proceso de producción (Pozo, J. 2016).

\section{Materiales y método}

\section{Descripción del área en studio}

La zona de estudio se encuentra ubicada en el cantón Loreto, provincia de Orellana. Sus coordenadas de localización son: latitud Sur $0^{\circ} 44^{\prime}$ y longitud oeste $77^{\circ} 27^{\prime}$, con una altitud de 450 m.s.n.m. Sus condiciones climatológicas medias anuales son temperatura $25{ }^{\circ} \mathrm{C}$, precipitación $3500 \mathrm{~mm} /$ año y una humedad relativa del $90 \%$. Según la clasificación ecológica de Holdrige la zona en estudio corresponde a la formación ecológica Bosque Húmedo Tropical (b.h.T.). El suelo de topografía plana está clasificado como Oxic Dystrudepts (Sánchez, 2017), de textura franco arcilloso y una estructura de bloques angulares.

\section{Población y muestra}

Debido a que la investigación busca establecer la relación entre la utilización de fertilizantes y la calidad del suelo. Se inicia con la búsqueda de los fertilizantes más utilizados en la zona en el cultivo de maíz, para lo cual se realiza un sondeo mediante encuesta, la estimación de la muestra se realiza con un universo finito.

$$
n=\frac{N * Z_{a}^{2} * p * q}{d^{2} *(N-1)+Z_{a}^{2} * p * q}
$$

Donde, el tamaño de la población es de 271 agricultores de maíz en el cantón, el nivel de confianza (Z) de 1,96; probabilidad de éxito (p) 0,9 y una precisión (D) de 0,05. Con un total de 92 encuestas.

\section{Muestreo experimental}

Para determinar el número mínimo de muestras, observaciones o réplicas efectuadas en el presente estudio se calculó utilizando la formula.

$$
n=\frac{W-W^{2} * Z_{\beta}+1.4 Z_{\alpha}^{2}}{W^{2}}
$$

Se plantea que los resultados del experimento deberán tener una significación $(\alpha)$ de $0,05(5 \%)$ que corresponde a un nivel de confianza (1- $\alpha)$ de1 95\% (0.95), se observa que, para este nivel de confianza, $Z \alpha$ tiene un valor de 1,96; para un valor estadístico $\beta$ igual a $0,10(90 \%)$, existe un $Z \beta$ de 1 , y la diferencia mínima observable (W) es de 0,3 ; dando como resultado un total de 6 muestras. 


\section{Diseño de parcelas}

Para el estudio del suelo se realizó la aleatorización de las parcelas de $200 \mathrm{~m}^{2}$ cada una.

Tabla 1. Distribución de las parcelas
\begin{tabular}{|c|c|c|c|}
\hline T1 & P 3 & P 2 & P1 \\
\hline T2 & P2 & P1 & P3 \\
\hline T3 & P1 & P 3 & P 2 \\
\hline
\end{tabular}

En la Tabla 1 se muestra la distribución de las parcelas en estudio donde; T1:18 días después de la siembra, 8g/planta de 10-30-10, a $10 \mathrm{~cm}$ de la planta; T2: 28 días después de la siembra, $20 \mathrm{gr} / \mathrm{planta}$ de urea, a $30 \mathrm{~cm}$ de la planta; y, T3 38 días después de la siembra, $30 \mathrm{gr} / \mathrm{planta}$ de urea, a $30 \mathrm{~cm}$ de la planta.

\section{Toma de muestras}

La toma de las muestras se realizó 8 días después de aplicar el tratamiento en las parcelas utilizando el método de zig-zag.

\section{Análisis}

La determinación de $\mathrm{pH}$ se lo realizó con potenciómetro en relación suelo: agua de 1:2,5; el Nitrógeno amoniacal (NH4) por el método de Kjeldhal; Fosforo (P), Potasio (K), se determinaron por Olsen modificado con $\mathrm{pH}$ 8.5; la suma de Bases Cambiables (Calcio, Magnesio) con la extracción de $\mathrm{CH} 3 \mathrm{COONH} 41 \mathrm{~N} \mathrm{pH} 7$ en pasta satura de suelo leída en el espectrofotómetro. La determinación de la velocidad de infiltración se realizó a través de infiltración metros de doble anillo (dos cilindros concéntricos) y la materia orgánica se determinó mediante la tasa de liberación de $\mathrm{CO}^{2}$. Para medir la respiración potencial de los organismos del suelo. La temperatura del suelo a $5 \mathrm{~cm}$ de profundidad.

\section{Identificación de variables}

En el caso de estudio se utilizó experimentos simples estudiando un solo factor de variación (Indicadores); siendo los indicadores: la temperatura, nivel $\mathrm{pH}$, textura, infiltración, Nitrógeno $\left(\mathrm{NH}^{+}\right)$, fósforo $(\mathrm{P})$, potasio $(\mathrm{K})$, calcio $(\mathrm{Ca})$ y magnesio $(\mathrm{Mg})$, materia orgánica $(\mathrm{MO}) \mathrm{y}$, la variable dependiente es la calidad de suelo.

\section{Análisis estadísticos}

Se analizó cada uno de los factores independientemente por lo que se aplicó un "Diseño de un modelo con efectos fijos".

\section{Índices de calidad de suelos}

Se realiza un análisis comparativo del resultado de índices (Cantú, 2007). 


\section{Resultados y Discusión}

En la figura 1 se muestra los resultados de la encuesta realizada a los productores de maíz del cantón Loreto donde se puede observar que en la preparación del suelo se utiliza con mayor frecuencia la "Urea" con un 36,61\% y " $10-30-10$ " con un porcentaje de $36,61 \%$, en su mayoría los productores realizan esta fertilización de manera empírica.

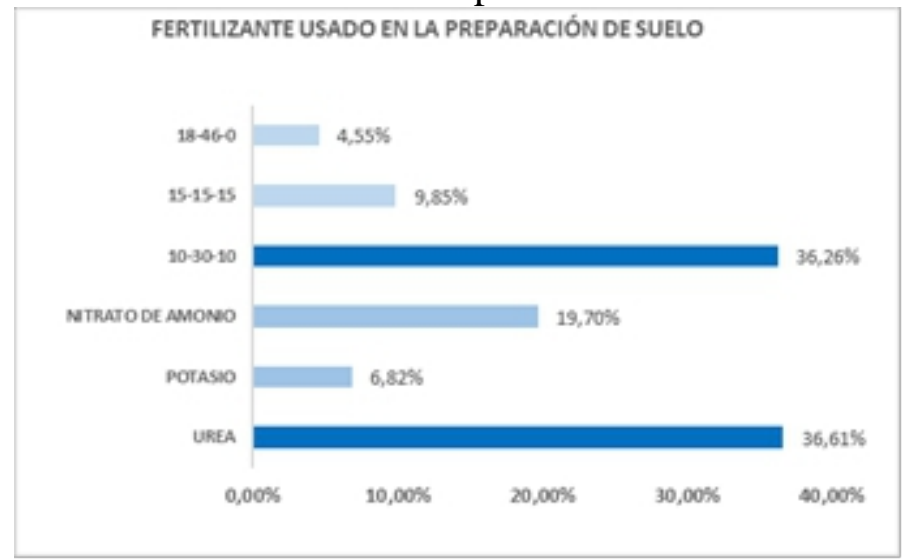

Figura 1. Tipo de fertilizantes usados en la preparación de suelo para el cultivo de maíz.

Tabla 2. Resultados análisis experimental modelo con efectos fijos.

\begin{tabular}{|c|c|c|c|c|c|c|c|c|}
\hline \multirow[t]{2}{*}{ Variables } & \multicolumn{6}{|c|}{ Tratamiento } & \multirow[t]{2}{*}{ E.E } & \multirow[t]{2}{*}{ Prob. } \\
\hline & T1 & & T2 & & T3 & & & \\
\hline Temperatura $\left({ }^{\circ} \mathbf{C}\right)$ & 25,50 & $\mathrm{a}$ & 25,57 & $\mathrm{a}$ & 25,70 & A & 0,18 & 0,74 \\
\hline $\mathbf{P h}$ & 6,22 & $\mathrm{a}$ & 6,24 & $\mathrm{a}$ & 6,40 & A & 0,11 & 0,50 \\
\hline Infiltración (cm/min) & 0,112 & $\mathrm{a}$ & 0,109 & $\mathrm{a}$ & 0,106 & $\mathrm{~A}$ & 0,06 & 0,99 \\
\hline Materia orgánica (g) & 4.016 & $\mathrm{a}$ & 4,004 & $\mathrm{a}$ & 4,005 & A & 0.06 & 0,06 \\
\hline Nitrógeno $\mathrm{NH}^{+}(\mathrm{g})$ & 47,83 & $\mathrm{a}$ & 33,97 & $\mathrm{~b}$ & 30,17 & B & 2,58 & 0,01 \\
\hline Fósforo (g) & 16,03 & $\mathrm{a}$ & 11,87 & $\mathrm{~b}$ & 10,20 & B & 0,67 & 0,00 \\
\hline Potasio (g) & 0,17 & $\mathrm{a}$ & 0,13 & $\mathrm{~b}$ & 0,10 & $\mathrm{C}$ & 0,01 & 0,00 \\
\hline Calcio (g) & 13,27 & $\mathrm{a}$ & 12,96 & $\mathrm{a}$ & 9,88 & A & 0,82 & 0,05 \\
\hline Magnesio (g) & 1,24 & $\mathrm{a}$ & 1,05 & $a b$ & 0,69 & B & 0,11 & 0,05 \\
\hline
\end{tabular}

Fuente: Resultados análisis experimental

Elaborado por: Brito Marcela

En la Tabla 2, se observa diferencia significativa en los tratamientos estudiados para los indicadores nitrógeno $\left(\mathrm{NH}^{+}\right)$, fósforo $(\mathrm{P})$, y potasio $(\mathrm{K})$, con $(\mathrm{p}>0,01)$.

En el caso del nitrógeno en los tratamientos T2 Y T3 en los que se aplicó urea la reducción es más evidente esto debido a que según (FERTINOVA, 2010), La Urea se hidroliza con rapidez por efecto de la enzima "ureasa" y por la temperatura del suelo, al hidrolizarse produce Amonio y bicarbonato, una vez que la urea se ha convertido en Amonio, éste es absorbido por las arcillas y la materia orgánica del suelo, los bicarbonatos reaccionan favoreciendo el incremento de $\mathrm{pH}$. En la teoría se menciona que 
este incremento desplaza el equilibrio del amonio y amoníaco favoreciendo la volatilización a la atmósfera (Sainz Rozas, 1997), en el caso del T1 del fertilizante 10-30-10 al aplicar menos cantidad de nitrógeno este tiene la posibilidad de fijarse en la materia orgánica y arcillas antes de volatilizarse.

En el parámetro $\mathrm{P}$ (fosforo), y K (potasio) en el T1, se obtuvo mejores resultados esto debido a que al añadir el fertilizante compuesto se aporta NPK en una proporción de 10\%-30\%-10\% respectivamente; mientras que en el T2 y T3 el aporte es de 46\% de nitrógeno sin aportación de fósforo y Potasio. Investigaciones llevadas a cabo durante la década del 80 demostraron que relaciones significativas entre el nivel de fosforo disponible a la siembra y la respuesta a la fertilización fosfatada en el del maíz de tal modo que el fosforo esté disponible para el cultivo desde la siembra. La reducida movilidad del ión ortofosfato y la retención del fertilizante fosfatado en el suelo requiere de la aplicación localizada del mismo (Ferreira, 2018).

En el caso del potasio es importante resaltar el balance $\mathrm{N}: \mathrm{K}$ desde los estadios iniciales de desarrollo del cultivo. Según (Loue, 1978) Este balance tiene mucha influencia sobre el acame, ya que altos contenidos de $\mathrm{N}$ con bajos contenidos de $\mathrm{K}$ favorecen el volcamiento de las plantas de maíz.

Los indicadores temperatura, $\mathrm{pH}$, infiltración, calcio y magnesio no presentan variación significativa en los valores de los tres tratamientos. 
Tabla 3. Correlación general de datos de los índices de calidad.

\begin{tabular}{|c|c|c|c|c|c|c|c|c|c|c|}
\hline & & Var. ${ }^{\circ} \mathrm{C}$ & Var. pH & Var. Infiltración & $\begin{array}{c}\text { Var. } \\
\text { Amoniaco }\end{array}$ & $\begin{array}{c}\text { Var. } \\
\text { Fósforo }\end{array}$ & Var. Potasio & $\begin{array}{c}\text { Var. } \\
\text { Calcio }\end{array}$ & Var. Magnesio & $\begin{array}{c}\text { Var. Mat } \\
\text { Orgánica } \\
\end{array}$ \\
\hline Var. ${ }^{\circ} \mathrm{C}$ & $\begin{array}{c}\text { Correlación de Pearson } \\
\text { Sig. (bilateral) } \\
\text { N }\end{array}$ & $\begin{array}{l}1 \\
9 \\
\end{array}$ & $\begin{array}{r}0,114 \\
0,769 \\
9 \\
\end{array}$ & $\begin{array}{r}-0,129 \\
0,740 \\
9 \\
\end{array}$ & $\begin{array}{r}-0,031 \\
0,936 \\
9 \\
\end{array}$ & $\begin{array}{r}-0,260 \\
0,500 \\
9 \\
\end{array}$ & $\begin{array}{r}0,320 \\
0,401 \\
9 \\
\end{array}$ & $\begin{array}{r}0,278 \\
0,468 \\
9 \\
\end{array}$ & $\begin{array}{r}-0,077 \\
0,843 \\
9 \\
\end{array}$ & $\begin{array}{r}0,432 \\
0,245 \\
9 \\
\end{array}$ \\
\hline Var. pH & $\begin{array}{c}\text { Correlación de Pearson } \\
\text { Sig. (bilateral) } \\
\mathrm{N} \\
\end{array}$ & $\begin{array}{r}0,114 \\
0,769 \\
9 \\
\end{array}$ & $\begin{array}{l}1 \\
9 \\
\end{array}$ & $\begin{array}{r}0,207 \\
0,593 \\
9 \\
\end{array}$ & $\begin{array}{r}-0,375 \\
0,320 \\
9 \\
\end{array}$ & $\begin{array}{r}0,055 \\
0,887 \\
9 \\
\end{array}$ & $\begin{array}{r}-0,118 \\
0,761 \\
9 \\
\end{array}$ & $\begin{array}{r}-0,126 \\
0,747 \\
9 \\
\end{array}$ & $\begin{array}{r}-, 791 * \\
0,011 \\
9 \\
\end{array}$ & $\begin{array}{r}0,432 \\
0,245 \\
9 \\
\end{array}$ \\
\hline Var. Infiltración & $\begin{array}{c}\text { Correlación de Pearson } \\
\text { Sig. (bilateral) } \\
\mathrm{N} \\
\end{array}$ & $\begin{array}{r}-0,129 \\
0,740 \\
9 \\
\end{array}$ & $\begin{array}{r}0,207 \\
0,593 \\
9 \\
\end{array}$ & 9 & $\begin{array}{r}0,352 \\
0,353 \\
9 \\
\end{array}$ & $\begin{array}{r}, 739 * \\
0,023 \\
9 \\
\end{array}$ & $\begin{array}{r}-0,127 \\
0,746 \\
9 \\
\end{array}$ & $\begin{array}{r}0,432 \\
0,245 \\
9 \\
\end{array}$ & $\begin{array}{r}-0,253 \\
0,511 \\
9 \\
\end{array}$ & $\begin{array}{r}-0,031 \\
0,996 \\
9 \\
\end{array}$ \\
\hline Var. Amoniaco & $\begin{array}{c}\text { Correlación de Pearson } \\
\text { Sig. (bilateral) } \\
\mathrm{N} \\
\end{array}$ & $\begin{array}{r}-0,031 \\
0,936 \\
9 \\
\end{array}$ & $\begin{array}{r}-0,375 \\
0,320 \\
9 \\
\end{array}$ & $\begin{array}{r}0,352 \\
0,353 \\
9 \\
\end{array}$ & 9 & $\begin{array}{r}0,291 \\
0,448 \\
9 \\
\end{array}$ & $\begin{array}{r}0,185 \\
0,633 \\
9 \\
\end{array}$ & $\begin{array}{r}, 707 * \\
0,033 \\
9 \\
\end{array}$ & $\begin{array}{r}0,002 \\
0,997 \\
9 \\
\end{array}$ & $\begin{array}{r}0,185 \\
0,633 \\
9 \\
\end{array}$ \\
\hline Var. Fósforo & $\begin{array}{c}\text { Correlación de Pearson } \\
\text { Sig. (bilateral) } \\
\mathrm{N} \\
\end{array}$ & $\begin{array}{r}-0,260 \\
0,500 \\
9 \\
\end{array}$ & $\begin{array}{r}0,055 \\
0,887 \\
9 \\
\end{array}$ & $\begin{array}{r}, 739 * \\
0,023 \\
9 \\
\end{array}$ & $\begin{array}{r}0,291 \\
0,448 \\
9 \\
\end{array}$ & 9 & $\begin{array}{r}0,452 \\
0,222 \\
9 \\
\end{array}$ & $\begin{array}{r}0,505 \\
0,165 \\
9 \\
\end{array}$ & $\begin{array}{r}-0,311 \\
0,416 \\
9 \\
\end{array}$ & $\begin{array}{r}0,352 \\
0,023 \\
9 \\
\end{array}$ \\
\hline Var. Potasio & $\begin{array}{c}\text { Correlación de Pearson } \\
\text { Sig. (bilateral) } \\
\mathrm{N} \\
\end{array}$ & $\begin{array}{r}0,320 \\
0,401 \\
9 \\
\end{array}$ & $\begin{array}{r}-0,118 \\
0,761 \\
9 \\
\end{array}$ & $\begin{array}{r}-0,127 \\
0,746 \\
9 \\
\end{array}$ & $\begin{array}{r}0,185 \\
0,633 \\
9 \\
\end{array}$ & $\begin{array}{r}0,452 \\
0,222 \\
9 \\
\end{array}$ & 1 & $\begin{array}{r}0,540 \\
0,134 \\
9 \\
\end{array}$ & $\begin{array}{r}-0,273 \\
0,478 \\
9 \\
\end{array}$ & $\begin{array}{r}-0,126 \\
0,747 \\
9 \\
\end{array}$ \\
\hline Var. Calcio & $\begin{array}{c}\text { Correlación de Pearson } \\
\text { Sig. (bilateral) } \\
\mathrm{N}\end{array}$ & $\begin{array}{r}0,278 \\
0,468 \\
9 \\
\end{array}$ & $\begin{array}{r}-0,126 \\
0,747 \\
9 \\
\end{array}$ & $\begin{array}{r}0,432 \\
0,245 \\
9 \\
\end{array}$ & $\begin{array}{r}, 707 * \\
0,033 \\
9 \\
\end{array}$ & $\begin{array}{r}0,505 \\
0,165 \\
9 \\
\end{array}$ & $\begin{array}{r}0,540 \\
0,134 \\
9 \\
\end{array}$ & 9 & $\begin{array}{r}-0,391 \\
0,299 \\
9 \\
\end{array}$ & $\begin{array}{r}-0,031 \\
0,500 \\
9 \\
\end{array}$ \\
\hline Var. Magnesio & $\begin{array}{c}\text { Correlación de Pearson } \\
\text { Sig. (bilateral) } \\
\mathrm{N}\end{array}$ & $\begin{array}{r}-0,077 \\
0,843 \\
9 \\
\end{array}$ & $\begin{array}{r}-, 791 * \\
0,011 \\
9 \\
\end{array}$ & $\begin{array}{r}-0,253 \\
0,511 \\
9 \\
\end{array}$ & $\begin{array}{r}0,002 \\
0,997 \\
9 \\
\end{array}$ & $\begin{array}{r}-0,311 \\
0,416 \\
9 \\
\end{array}$ & $\begin{array}{r}-0,273 \\
0,478 \\
9 \\
\end{array}$ & $\begin{array}{r}-0,391 \\
0,299 \\
9 \\
\end{array}$ & 1 & $\begin{array}{r}-0,311 \\
0,299 \\
9 \\
\end{array}$ \\
\hline Var. Mat Orgánica & $\begin{array}{c}\text { Correlación de Pearson } \\
\text { ig. (bilateral) } \\
\mathrm{N}\end{array}$ & $\begin{array}{r}0,114 \\
9\end{array}$ & $\begin{array}{r}0,320 \\
0,887 \\
9\end{array}$ & $\begin{array}{r}-0,118 \\
0,023 \\
9\end{array}$ & $\begin{array}{r}-0,127 \\
0,320 \\
9\end{array}$ & $\begin{array}{r}0,185 \\
0,887 \\
9\end{array}$ & $\begin{array}{r}0,207 \\
0,761 \\
9\end{array}$ & $\begin{array}{r}-0,375 \\
0,747 \\
9\end{array}$ & $\begin{array}{r}0,055 \\
0,416 \\
9\end{array}$ & 1 \\
\hline
\end{tabular}


Tabla 4. Evaluación de Indicadores de calidad de suelo por tratamiento

\begin{tabular}{|c|c|c|c|c|c|c|c|}
\hline \multirow[b]{2}{*}{ INDICADORES } & \multicolumn{2}{|c|}{ T1 } & \multicolumn{2}{|c|}{$\mathbf{T 2}$} & \multicolumn{2}{|r|}{ T3 } & \\
\hline & VALOR & $\begin{array}{c}\text { INDICE } \\
\text { DE } \\
\text { CALIDAD } \\
\end{array}$ & VALOR & $\begin{array}{c}\text { INDICE } \\
\text { DE } \\
\text { CALIDAD } \\
\end{array}$ & VALOR & $\begin{array}{c}\text { INDICE } \\
\text { DE } \\
\text { CALIDAD } \\
\end{array}$ & \\
\hline TEMPERATURA & 25,50 & 0,80 & 25,57 & 0,80 & 25,70 & 0,80 & $\begin{array}{l}\text { Temperatura } \\
\text { óptima para } \\
\text { suelos de } \\
\text { clima cálido }\end{array}$ \\
\hline $\mathbf{p H}$ & 6,22 & 0,80 & 6,24 & 0,80 & 6,40 & 0,80 & $\begin{array}{c}\text { el nivel de } \\
\text { pH se } \\
\text { encuentra } \\
\text { alrededor de } \\
\text { la neutralidad }\end{array}$ \\
\hline INFILTRACION & 0,11 & 0,30 & 0,11 & 0,30 & 0,11 & 0,30 & muy lenta \\
\hline NITROGENO TOTAL & 38,33 & 0,40 & 40,47 & 0,40 & 33,27 & 0,40 & $\begin{array}{c}\text { El nivel de } \\
\text { NH4 se } \\
\text { encuentra en } \\
\text { un nivel bajo }\end{array}$ \\
\hline FOSFORO & 11,93 & 0,30 & 12,40 & 0,60 & 13,77 & 0,60 & $\begin{array}{l}\text { El máximo } \\
\text { nivel de } \\
\text { Fosforo que } \\
\text { se puede } \\
\text { encontrar es } \\
\text { de } 30 \mathrm{ppm} .\end{array}$ \\
\hline POTASIO & 0,10 & 0,10 & 0,15 & 0,15 & 0,15 & 0,15 & $\begin{array}{c}\text { El nivel de } \\
\text { potasio es } \\
\text { bajo cuando } \\
\text { es menor a } \\
0,10 \\
\text { meq } / 100 \mathrm{ml} \text {. }\end{array}$ \\
\hline CALCIO & 9,88 & 0,40 & 12,81 & 0,40 & 13,09 & 0,40 & $\begin{array}{l}\text { El indicador } \\
\text { es alto de } \\
\text { calcio igual o } \\
\text { superior a } 40 \\
\text { meq } / 100 \mathrm{ml}\end{array}$ \\
\hline MAGNESIO & 1,21 & 0,40 & 0,92 & 0,40 & 0,84 & 0,40 & $\begin{array}{c}\text { Un valor } \\
\text { optimo un } \\
\text { nivel de } 10 \text {, }\end{array}$ \\
\hline MATERIA ORGANICA & 4,02 & 0,80 & 4,00 & 0,80 & 3,99 & 0,80 & $\begin{array}{l}\text { la Materia } \\
\text { orgánica se } \\
\text { encuentra en } \\
\text { niveles altos }\end{array}$ \\
\hline TOTAL & & $\mathbf{0 , 4 8}$ & & $\mathbf{0 , 5 2}$ & & $\mathbf{0 , 5 2}$ & \\
\hline
\end{tabular}

Fuente: (Villar, 20016)

Tabla 5. Índice de calidad de suelos

\begin{tabular}{ccc}
\hline Índice de calidad de suelos & Escala & Clases \\
\hline Muy alta calidad & $0,80-1,00$ & 1 \\
Alta Calidad & $0,60-0,70$ & 2 \\
Moderada calidad & $0,40-0,59$ & 3 \\
Baja Calidad & $0,20-0,39$ & 4 \\
Muy Baja Calidad & $0,00-0,19$ & 5 \\
\hline
\end{tabular}

Fuente: Cantu, 2017 


\section{Conclusión}

Tanto los niveles y tipos de fertilizantes como la época de aplicación utilizados en el suelo del cultivo de maíz en el cantón Loreto no modifican los índices de calidad en los indicadores pH, materia orgánica, temperatura, infiltración, calcio y magnesio. Las diferencias significativas observadas en los tratamientos en estudio fueron para los indicadores nitrógeno $\left(\mathrm{NH} 4^{+}\right)$, fósforo $(\mathrm{P})$, y potasio $(\mathrm{K})$; esto debido a que los fertilizantes utilizados por los agricultores Urea y 10-30-10, aportan los macronutrientes en diversa proporción y estos son utilizados por el cultivo para su desarrollo. Es importante tomar en cuenta la necesidad de realizar análisis de los suelos para poder tomar la decisión de que tipo y dosis de fertilizante son las más adecuadas para el cultivo, así como las épocas ideales de aplicación según los requerimientos fenológicos de la planta y la calidad ambiental que el suelo presente.

\section{References:}

1. Astier-Calderón, M. M. (2002). Derivación de indicadores de calidad de suelos en el contexto de la agricultura sustentable. Agrociencia, 605 -610 .

2. Cantú, M. B. (2007). Evaluación de la Calidad de suelos mediante el uso de Indicadores e Indices. Cielo.

3. Dumanski, J. G. (1998). Indicators of land quality and sustainable land management. TheWorld Bank.

4. Ferreira, G. T. (2018). Effect of corn planting population on phosphorus concentration and uptake in corn silage. Dairy Science.

5. Ferreras, L., Toresani, S., Bonel, B., Fernández, E., Bacigaluppo, S., Faggioli, V., \& Beltrán, C. (2009). Parámetros químicos y biológicos como indicadores de calidad del suelo en diferentes manejos. Ciencia del suelo , 103-114.

6. FERTINOVA. (2010). Ficha Técnica Urea 46-0-0. Obtenido de http://www.fertinova.mx/sites/default/files/FICHA\%20UREA.pdf

7. Garcia, F. (2016). Fósforo y Azufre en el Cultivo de Maíz. Fertilizando.

8. García, Y. R. (2012). Indicadores de la calidad de los suelos: una nueva manera de evaluar este recurso . Pastos y Forrajes, 125-138.

9. Gregorich, E. (1994). Towards a minimum data set to asses soil organic matter quality in agricultural soils. Canadian J. of Soil Science, 74-367.

10. Havlin, J. e. (1999). Soil fertility and fertilizers: an introduction to nutrient management. New Jersey, USA.: Prentice Hall.

11. Loue, A. (1978). 11 Congress International of the Potash Institute. Nitrogen Relation Potassium in corn culture, (págs. 407-33). Bern. 
12. Moreno, C. G. (2015). INFLUENCIA DEL MANEJO SOBRE LA CALIDAD DEL SUELO. ECUADOR ES CALIDAD: Revista Científica Ecuatoriana, 2, 33-40.

13. PNA Potassium Nitrate Association. (2018). Boletin PNA 2017-01. Obtenido de http://www.kno3.org/images/pna/pdf/html_pna_nl_es_1_16_2.pdf

14. Pozo, J. (2014). Informe rubro maiz - Provincia de Orellana. MAGAP.

15. Ross, S. M. (1993). Organic matter in tropical soils: current conditions, concerns and prospects for conservation. Progress in Physical Geography, 265-305.

16. Sainz Rozas, H. E. (1997). Volatilización de amoníaco desde urea aplicada al cultivo de maíz bajo siembra directa. Ciencia del Suelo 15, 12-16.

17. Sánchez, D. M. (2017). Soils from de Amazonia. En J. M. Espinosa, Soils of Ecuador.

18. Soto, G. (2006). Calidad de los suelos: una nueva visión del suelo. I Congreso Latinoamericano de Experimentadores e Investigadores en Producción Orgánica. Managua - Nicaragua.

19. Villar, P. V. (20016). GUIA DE LA FERTILITAT DELS SÒLS I LA NUTRICIÓ VEGETAL EN PRODUCCIÓ INTEGRADA. Cataluña. 